\title{
Risk Factors for In-Hospital Complications of Fall-Related Fractures among Older Chinese: A Retrospective Study
}

\author{
Hong-Ying Pi, ${ }^{1}$ Yuan Gao, ${ }^{2}$ Jing Wang, ${ }^{1}$ Meng-Meng Hu, ${ }^{3}$ Dan Nie, ${ }^{4}$ and Pei-Pei Peng ${ }^{5}$ \\ ${ }^{1}$ Department of Nursing, Chinese PLA General Hospital, No. 28 Fuxing Road, Haidian District, Beijing 100853, China \\ ${ }^{2}$ Department of Orthopedics, Chinese PLA General Hospital, No. 28 Fuxing Road, Haidian District, \\ Beijing 100853, China \\ ${ }^{3}$ Department of Medical Oncology, Nanlou Faculty of Clinical Medicine, Chinese PLA General Hospital, \\ No. 28 Fuxing Road, Haidian District, Beijing 100853, China \\ ${ }^{4}$ Department of Digestive Medicine, Nanlou Faculty of Clinical Medicine, Chinese PLA General Hospital, \\ No. 28 Fuxing Road, Haidian District, Beijing 100853, China \\ ${ }^{5}$ The 15th Battalion of Student Brigade, Third Military Medical University, No. 30 Gaotanyan Main Street, \\ Shapingba District, Chongqing 400038, China \\ Correspondence should be addressed to Hong-Ying Pi; venusruby@foxmail.com
}

Received 15 July 2016; Accepted 7 December 2016

Academic Editor: Zhiyong Hou

Copyright (C) 2016 Hong-Ying Pi et al. This is an open access article distributed under the Creative Commons Attribution License, which permits unrestricted use, distribution, and reproduction in any medium, provided the original work is properly cited.

\begin{abstract}
Purpose. The aim of this study was to investigate the risk factors and the efficacy of the preventive measurements for the in-hospital complications of fall-related fractures. Methods. The data on older Chinese patients with fall-related fractures were collected, including information on the patients, diseases, and preventive measurements. The potential risk factors for the in-hospital complications included health status on admission, comorbidity, fractures, preventive measures of the complications, and drugs use for the comorbidity. After univariate analyses, multivariate logistic regression analyses were applied to investigate the impact of the potential risk factors on the number of the complications and each individual complication, respectively, and the efficacy of the preventive measurements. Results. A total of 525 male and 1367 female were included in this study. After univariate analyses, multiple logistic regression showed that dementia, pneumonia, antidepressant, postural hypotension, and cerebral infarction could increase the incidence and number of comorbidities. Meanwhile, dementia has shown the strongest association with each individual complication. Conclusions. Different combinations of comorbidity, medication use, and preventive measurements were related to the in-hospital complications of fall-related fractures. Dementia emerged as the most important risk factor for these complications, while most of the preventive measurements could not reduce their incidences.
\end{abstract}

\section{Introduction}

Falls are an important public health issue among older adults as they are one of the leading causes of fall-related injury and death in the populations [1-4]. Fall-related fracture is one of the most disabling problem of older patients and often trigger a downward spiral in their health which has a close association with the restriction of activity. That may eventually result in many long-term bedfast complications, including pressure ulcer, hospital-acquired pneumonia (HAP), urinary tract infection (UTI), and lower extremity venous thromboembolism (VTE), and even disability or deaths, especially in patients with comorbidity $[3,4]$.

Several studies have identified the characteristics and potential risk factors for fall-related fractures [1, 3-15]. For example, Sibley et al. [4] studied the relationship between falls and the number of chronic diseases and reported that $62 \%$ of fallers had multimorbidity, such as arthritis, visual impairment, hypertension, chronic obstructive pulmonary disease, diabetes, or heart disease, while $23.8 \%$ had a single chronic disease. Other studies also showed that comorbidity is an important factor for falls $[6,16]$. Some acute diseases 
and medication use, such as cardiovascular or psychotropic drugs, also have been considered the primary precipitating risk factors for falls $[6,17]$.

However, there are limited studies on risk factors and preventive measurements for in-hospital complications of fall-related fractures. This study is an attempt to identify the risk factors for in-hospital complications of fall-related fractures among older Chinese patients and if the preventive measurements used in the clinical activities could decrease the incidence of these in-hospital complications. We collected health and clinical characteristics on patients, including their comorbidity conditions, medication use, and other health statuses of the populations, and tried to explore the interactive relationships between these viable and preventive measurements and their impact on the incidences of inhospital complications.

\section{Materials and Methods}

2.1. Design and Patients. This retrospective study was conducted at Chinese PLA General Hospital and approved by the hospital's Ethics and Institutional Review Committees (Code: S2014-055-07). The waiver of patients' informed consent was granted due to the retrospective nature and anonymous patients data of the study. Data were obtained on old Chinese patients with fall-related fractures who were admitted to the Department of Orthopedics and Trauma at the Chinese PLA General Hospital between April 2004 and July 2014. Eligible patients were those aged 60 years or older who had fall-related fractures on admission.

Patients who were admitted with high energy fractures caused by traffic accident or high falling were excluded from the study. Patients who were transferred from other hospitals were also excluded.

2.2. Information on the Patients and Diseases. Information on the patients and injuries was obtained from the electrical medical records. Six types of in-hospital complications were noted, including pressure ulcer, HAP, UTI, lower extremity VTE, constipation, foot drop, and incision complications. The potential risk factors for the in-hospital complications of fallrelated fractures were classified as the following five categories: physical status on admission, comorbidities, fracture sites, preventive measures of the in-hospital complications, and drugs use for the comorbidities. Two individual doctors collected half of all the data in an independent manner and checked it with each other after entire collection.

The physical status on admission includes nutritional status, conscious status, and sensory impairment. The nutritional status is assessed by using the short-form MiniNutritional Assessment and classified as three grades (malnourished, moderate/at risk of malnutrition, and normal) according to the protocol [18]. The conscious status is classified as five grades, including normal, confusion, delirium, somnolence, and coma, in order of decreased level. Sensory impairment is defined as hearing or vision impairment. The comorbidities include cardiovascular diseases (hypertension, coronary heart disease, postural hypotension, atrial fibrillation, ventricular tachycardia, supraventricular tachycardia, atrial flutter, atrioventricular block, sick sinus syndrome, ventricular premature beat, acute myocardial infarction, rheumatic heart disease, sinus bradycardia, hyperlipemia, etc.), central nervous system (CNS) diseases (cerebral infarction, dementia, cerebral hemorrhage, transient ischemic attack, Parkinson's disease, myasthenia gravis, epilepsy, etc.), bone and joint diseases (osteoarthritis, osteoporosis, protrusion of intervertebral disc, pathological fracture, bone hyperplasia, cervical spondylosis, etc.), respiratory system diseases (pneumonia, chronic obstructive pulmonary disease, etc.), diabetes, cataract, and anemia. In addition, skin problems were also noted, including broken skin, skin yellowing, hematocyanosis, rash, hyperpigmentation, ochrodermia, edema, dehydration, furuncle, petechia, bruise or blood spot, scar, and pale skin.

The fracture site includes radial fracture, femoral neck fracture, femoral intertrochanteric fracture, femoral shaft fracture, spinal fracture, and minor fracture, and the characteristics of fracture are classified as upper extremity, lower extremity, and spinal or multiple fractures.

2.3. Preventive Measures of In-Hospital Complications. In our clinical activities, several preventive measures of complications were used as a routine nursing work and performed on the included patients. The number and combination of the preventive measures performed were dependent on different attending doctors' decision based on the condition of each patient.

The preventive measures of pressure ulcer include (1) replacing the clothes and sheets regularly and keeping the bed clean and dry; (2) keeping the crissum and entire skin clean and dry; (3) turning patients over regularly; (4) providing patients dietary guidance; (5) other preventive measures.

The preventive measures of HAP include (1) guiding patients to deep breathing and coughing exercises; (2) assisting patients to regularly change the posture; (3) turning patients over and knocking back regularly; (4) atomization inhalation treatment; (5) other preventive measures.

The preventive measures of UTI include (1) drinking plenty of water; (2) disinfecting the urinary catheter regularly; (3) clipping the urinary catheter intermittently and instructing patients to strengthen the urinary sphincter with bladder training exercises; (4) replacing the urine bag and catheter regularly; (5) bladder irrigation regularly; (6) other preventive measures.

The preventive measures of lower extremity VTE include (1) guiding patients with lower limb functional exercise regularly; (2) wearing antithrombotic pressure belt with double lower limbs; (3) anticoagulant drugs use; (4) other preventive measures.

The preventive measures of constipation include (1) eating high-fiber foods; (2) taking abdominal massage regularly; (3) using glycerine enema or other laxatives; (4) other preventive measures.

2.4. Statistical Analysis. After proving normal distribution of the data, the mean differences of the continuous data between groups were compared by independent samples 
TABLE 1: The patients' general information.

\begin{tabular}{|c|c|c|c|c|c|}
\hline & Male $(N=525$, mean \pm SD $)$ & Female $(N=1367$, mean \pm SD $)$ & $p$ & Total $(N=1892$, mean \pm SD $)$ & Range \\
\hline Age (years) & $77.31 \pm 8.16$ & $75.74 \pm 8.7$ & $<0.001$ & $76.18 \pm 8.58$ & $60-101$ \\
\hline Hospital LOS (days) & $14.75 \pm 17.40$ & $11.68 \pm 24.39$ & 0.008 & $12.53 \pm 22.71$ & $0-615$ \\
\hline Grade 1 nursing (days) & $11.94 \pm 13.2$ & $9.19 \pm 21.26$ & 0.006 & $9.95 \pm 19.4$ & $0-527$ \\
\hline
\end{tabular}

$N$ : number; SD: standard deviation; LOS: length of stay.

t-test. Pearson's Chi square test and Fisher's exact test were used for detecting the interaction of two categorical variables. Kruskal-Wallis test was used for intergroup comparisons of three or multiple categorical variables. Correlations between categorical variables were determined using Spearman's correlation analysis. After univariate analyses, ordinal or multivariate analyses were applied to investigate the potential risk factors for the number of complications and each individual complication, respectively. The validity of the model was tested by Hosmer-Lemeshow statistic for goodness of fit. The hazard ratio of the potential risk factor was expressed as odds ratio (OR) values or regression coefficients $(\beta)$. A $p$ value less than 0.05 was considered statistically significant. All statistical procedures were accomplished with SPSS 20 software (SPSS Inc., Chicago, IL, USA).

\section{Results}

The population included are of a mean age of 76.18 years with 525 male and 1367 female. Both hospital length of stay (LOS) and length of grade 1 nursing are significantly longer in the male than female group. The general information is summarized in Table 1.

3.1. Comparison of the Health and Clinical Characteristics. Table 2 presents the comparison of the physical status and clinical characteristics between genders. It can be shown that the incidences of normal or malnourished nutritional status, femoral shaft fractures, thoracic vertebra fractures, and other minor fractures were significantly higher in female than male group with all $p$ values $<0.05$. The incidences of one or two CNS diseases, intertrochanteric fractures, and upper or lower extremity fractures were significantly higher in male than female group with all $p$ values $<0.05$. Spearman's correlation analysis shows that age has a significantly positive correlation with lower extremity fractures $(r=0.367, p<0.001)$ and negative correlations with upper extremity fractures $(r=$ $-0.361, p<0.001)$ and spinal fractures $(r=-0.128, p<$ $0.001)$. However, the negative association of age to spinal fractures only exists in female group $(r=-0.141, p<0.001)$.

There are also significantly positive correlations of the number of comorbidities to hospital length of stay $(r=0.141$, $p<0.001)$ and length of grade 1 nursing $(r=0.13, p<$ 0.001 ). Meanwhile, $48.57 \%$ of male and $46.65 \%$ of female patients have two comorbidities and over, although there is no significant difference of the number of comorbidities between genders.

The differences of the numbers of the preventive measures of the complications are also not consistent between genders
(Table 2). However, there are no significant differences of the number of the complications or the incidences of each individual complication between different genders groups (Table 3).

3.2. Univariate and Multivariate Analysis for the Factors of the Conscious Status. Pearson's Chi square test shows that intertrochanteric fracture, cerebral hemorrhage, cerebral infarction, dementia, other CNS diseases, diabetes, antidiabetic drug, antidepressant, pale skin, other skin diseases, constipation, sensory impairment, and nutritional status are risk factors for the conscious status (Table 4). Kruskal-Wallis one-way ANOVA shows that the age is significantly higher in the "confusion" group than in the "normal" group $(p<0.001)$ and higher in the "somnolence" group than in the "confusion" group $(p=0.024)$.

With the factors abovementioned as independent variables ordinal logistic regression also shows that age, nutritional status, dementia, and pale skin are independent risk factors for conscious status (Table 5).

Spearman's correlation analysis also shows that there are weak but significant positive correlations between conscious status and age $(r=0.112, p<0.001)$, nutritional status $(r=0.131, p<0.001)$, sensory impairment $(r=0.108$, $p<0.001)$, dementia $(r=0.154, p<0.001)$, and pale skin $(r=0.104, p<0.001)$.

3.3. Univariate and Multivariate Analysis for the Factors of Each Individual Complication. Pearson's Chi square test shows that femoral shaft fracture, broken skin, cerebral hemorrhage, dementia, turning over regularly, skin yellowing, rash, other skin problems, hypertension, and anemia are all risk factors for pressure ulcer. However, multivariate analysis shows that only femoral shaft fracture $(\mathrm{OR}=23.64, p=$ $0.023)$, broken skin ( $\mathrm{OR}=14.324, p=0.07)$, cerebral hemorrhage $(\mathrm{OR}=555.49, p=0.002)$, dementia $(\mathrm{OR}=$ $145.3, p=0.002)$, and turning over regularly $(\mathrm{OR}=0.039$, $p=0.017)$ are independent risk factors for pressure ulcer (Table 6).

Pearson's Chi square test shows that atrial fibrillation, cerebral infarction, dementia, changing posture regularly, and antihypertensive drugs are all risk factors for HAP. However, multivariate analysis excludes the risk of antihypertensive drugs (Table 7).

Univariate analysis of the risk of urinary tract infection shows that cerebral infarction, dementia, anemia, drinking more water, bladder irrigation, broken skin, skin yellowing, rash, and cerebral hemorrhage are all risk factors for UTI. However, multivariate logistic regression shows that only 
TABLE 2: Comparison of the physical status and characteristics of injuries between genders.

\begin{tabular}{|c|c|c|c|c|c|}
\hline Factors & Grade/N & Male/N (\%) & Female/N (\%) & $p$ & Total/N (\%) \\
\hline \multirow[t]{4}{*}{ Nutritional status } & & & & 0.036 & \\
\hline & Normal & $340(64.76)$ & $953(69.71)$ & 0.038 & $1293(68.34)$ \\
\hline & Moderate & $175(33.33)$ & $377(27.58)$ & 0.203 & $552(29.17)$ \\
\hline & Malnourished & $10(1.9)$ & $37(2.71)$ & 0.013 & $47(2.48)$ \\
\hline \multirow[t]{6}{*}{ Mental status } & & & & 0.433 & \\
\hline & Normal & $506(96.38)$ & $1320(96.56)$ & 0.726 & $1826(96.51)$ \\
\hline & Confusion & $18(3.43)$ & $44(3.22)$ & 0.818 & $62(3.27)$ \\
\hline & Delirium & $1(0.19)$ & 0 & 0.277 & $1(0.05)$ \\
\hline & Somnolence & 0 & $2(0.15)$ & 0.522 & $2(0.11)$ \\
\hline & Coma & 0 & $1(0.07)$ & 0.723 & 1 \\
\hline \multirow{2}{*}{ Sensory impairment } & No & $451(85.9)$ & $1216(88.95)$ & 0.067 & $1667(88.11)$ \\
\hline & Yes & $74(14.1)$ & $151(11.05)$ & 0.067 & 225 (11.89) \\
\hline \multirow[t]{5}{*}{ Dermal problems } & & & & 0.335 & \\
\hline & 0 & $126(24)$ & $347(25.38)$ & 0.534 & $473(25)$ \\
\hline & 1 & $388(73.9)$ & $1000(73.15)$ & 0.74 & $1388(73.36)$ \\
\hline & 2 & $10(1.9)$ & $20(1.46)$ & 0.307 & $30(1.59)$ \\
\hline & 3 & $1(0.19)$ & 0 & 0.277 & $1(0.05)$ \\
\hline \multirow[t]{6}{*}{ Cardiovascular diseases } & & & & 0.596 & \\
\hline & 0 & $260(49.52)$ & $630(46.09)$ & 0.18 & $890(47.04)$ \\
\hline & 1 & $199(37.7)$ & $534(39.06)$ & 0.589 & $733(38.74)$ \\
\hline & 2 & $61(11.62)$ & $186(13.61)$ & 0.251 & $247(13.05)$ \\
\hline & 3 & $5(0.95)$ & $16(1.17)$ & 0.685 & $21(1.11)$ \\
\hline & 4 & 0 & $1(0.07)$ & 0.723 & $1(0.05)$ \\
\hline \multirow[t]{5}{*}{ CNS diseases } & & & & $<0.001$ & \\
\hline & 0 & $367(69.9)$ & $1094(80.03)$ & $<0.001$ & $1461(77.22)$ \\
\hline & 1 & $137(26.1)$ & $246(18)$ & $<0.001$ & $383(20.24)$ \\
\hline & 2 & $19(3.62)$ & $23(1.68)$ & 0.011 & $42(2.22)$ \\
\hline & 3 & $2(0.38)$ & $4(0.29)$ & 0.53 & $6(0.32)$ \\
\hline \multirow[t]{5}{*}{ Bone and joint diseases } & & & & 0.187 & \\
\hline & 0 & $461(87.81)$ & $1185(86.69)$ & 0.515 & $1646(87)$ \\
\hline & 1 & $59(11.24)$ & $177(12.95)$ & 0.313 & $236(12.47)$ \\
\hline & 2 & $5(0.95)$ & $4(0.29)$ & 0.073 & $9(0.48)$ \\
\hline & 3 & 0 & $1(0.07)$ & 0.723 & $1(0.05)$ \\
\hline \multirow{2}{*}{ Respiratory system diseases } & 0 & $504(96)$ & $1317(96.34)$ & 0.408 & $1821(96.25)$ \\
\hline & 1 & $21(4)$ & $50(3.66)$ & 0.408 & $71(3.75)$ \\
\hline \multirow[t]{8}{*}{ Fracture site } & & & & $0.018^{\mathrm{a}}$ & \\
\hline & Radius & $61(11.6)$ & $154(11.3)$ & 0.828 & $215(11.36)$ \\
\hline & Femoral shaft & $23(4.4)$ & $91(6.7)$ & 0.037 & $114(6.3)$ \\
\hline & Femoral neck & $199(37.9)$ & $487(35.6)$ & 0.356 & $686(36.26)$ \\
\hline & Intertrochanteric & $196(37.3)$ & 409 (29.9) & 0.001 & $605(31.98)$ \\
\hline & Lumbar vertebra & $35(6.7)$ & $104(7.6)$ & 0.276 & $139(7.35)$ \\
\hline & Thoracic vertebra & 0 & $16(1.2)$ & 0.005 & $16(0.85)$ \\
\hline & Minor fracture & $26(5)$ & $131(9.6)$ & $<0.001$ & $157(8.3)$ \\
\hline \multirow[t]{5}{*}{ Fracture characteristics } & & & & 0.076 & \\
\hline & Upper extremity & $56(10.67)$ & $186(13.61)$ & 0.049 & $242(12.79)$ \\
\hline & Lower extremity & 409 (77.9) & $1000(73.15)$ & 0.019 & $1409(74.47)$ \\
\hline & Spine & $42(8)$ & $144(10.53)$ & 0.056 & $186(9.83)$ \\
\hline & Multiple & $18(3.43)$ & $37(2.71)$ & 0.243 & $55(2.91)$ \\
\hline
\end{tabular}


TABLE 2: Continued.

\begin{tabular}{|c|c|c|c|c|c|}
\hline Factors & Grade/N & Male/N (\%) & Female/N (\%) & $p$ & Total/N (\%) \\
\hline \multirow[t]{7}{*}{ Preventive measures of pressure ulcer } & & & & 0.001 & \\
\hline & 0 & $3(0.57)$ & $5(0.37)$ & 0.074 & $8(0.42)$ \\
\hline & 1 & $5(0.95)$ & $12(0.88)$ & 0.029 & $17(0.9)$ \\
\hline & 2 & $128(24.38)$ & $327(23.92)$ & $<0.001$ & $455(24.05)$ \\
\hline & 3 & $59(11.24)$ & $272(19.9)$ & 0.013 & $331(17.49)$ \\
\hline & 4 & $226(43.05)$ & $520(38.04)$ & $<0.001$ & $746(39.43)$ \\
\hline & 5 & $104(19.81)$ & $231(16.9)$ & 0.002 & $335(17.71)$ \\
\hline \multirow[t]{7}{*}{ Preventive measures of HAP } & & & & 0.009 & \\
\hline & 0 & 0 & $3(0.22)$ & 0.377 & $3(0.16)$ \\
\hline & 1 & $11(2.1)$ & $28(2.05)$ & 0.007 & $39(2.06)$ \\
\hline & 2 & $9(1.71)$ & $26(1.9)$ & 0.479 & $35(1.85)$ \\
\hline & 3 & $286(54.48)$ & $863(63.13)$ & 0.005 & $1149(60.73)$ \\
\hline & 4 & $191(36.38)$ & $399(29.19)$ & 0.004 & $590(31.18)$ \\
\hline & 5 & $28(5.33)$ & $48(3.51)$ & 0.016 & $76(4.02)$ \\
\hline \multirow[t]{8}{*}{ Preventive measures of UTI } & & & & 0.028 & \\
\hline & 0 & $3(0.57)$ & $11(0.8)$ & 0.01 & $14(0.74)$ \\
\hline & 1 & $106(20.19)$ & $365(26.7)$ & $<0.001$ & $471(24.89)$ \\
\hline & 2 & $18(3.43)$ & $62(4.54)$ & 0.364 & $80(4.23)$ \\
\hline & 3 & $100(19.05)$ & $251(18.36)$ & $<0.001$ & $351(18.55)$ \\
\hline & 4 & $269(51.24)$ & $592(43.31)$ & 0.402 & $861(45.51)$ \\
\hline & 5 & $28(5.33)$ & $85(6.22)$ & 0.482 & $113(5.97)$ \\
\hline & 6 & $1(0.19)$ & $1(0.07)$ & 0.522 & $2(0.11)$ \\
\hline \multirow[t]{6}{*}{ Preventive measures of lower extremity VTE } & & & & 0.058 & \\
\hline & 0 & $7(1.33)$ & $8(0.59)$ & 0.007 & $15(0.79)$ \\
\hline & 1 & $238(45.33)$ & $669(48.94)$ & $<0.001$ & $907(47.94)$ \\
\hline & 2 & $231(44)$ & $526(38.48)$ & $<0.001$ & $757(40.01)$ \\
\hline & 3 & $48(9.14)$ & $159(11.63)$ & $<0.001$ & $207(10.94)$ \\
\hline & 4 & $1(0.19)$ & $5(0.37)$ & 0.53 & $6(0.32)$ \\
\hline \multirow[t]{6}{*}{ Preventive measures of constipation } & & & & 0.628 & \\
\hline & 0 & $12(2.29)$ & $23(1.68)$ & $<0.001$ & $35(1.85)$ \\
\hline & 1 & $373(71.05)$ & $961(70.3)$ & 0.001 & $1334(70.51)$ \\
\hline & 2 & $137(26.1)$ & $378(27.65)$ & $<0.001$ & $515(27.22)$ \\
\hline & 3 & $3(0.57)$ & $4(0.29)$ & 0.621 & $7(0.37)$ \\
\hline & 4 & 0 & $1(0.07)$ & 0.723 & $1(0.05)$ \\
\hline
\end{tabular}

${ }^{a}$ Wilcoxon test; N: number; UTI: urinary tract infection; VTE: venous thromboembolism; HAP: hospital-acquired pneumonia; PU: pressure ulcer; CNS: central nervous system.

cerebral infarction, dementia, anemia, drinking more water, and bladder irrigation are independent risk factors for UTI. Meanwhile, the risk of UTI development can be significantly reduced by drinking more water $(\mathrm{OR}=0.013, p=0.021)$ but increased by bladder irrigation $(\mathrm{OR}=14.954, p=0.009)$ (Table 8).

Both univariate and multivariate analyses for the influencing factors of foot drop show that broken skin, cerebral infarction, dementia, and postural hypotension are independent risk factors for foot drop (Table 9).

3.4. Univariate and Multivariate Analysis for the Factors of the Complications Number. Pearson's Chi square test shows that the numbers of CNS diseases, respiratory system diseases, and dermal problems are significantly different between different groups of the complications number (Table 10). Spearman's correlation analysis shows that there are weak but significantly positive correlations between the complications number and the number of CNS diseases $(r=0.09, p<$ $0.001)$ and respiratory system diseases $(r=0.072, p<0.001)$, respectively. Kruskal-Wallis one-way ANOVA shows that the complications numbers are shown significantly greater in one or two CNS diseases groups than in no CNS disease group with $p$ values of 0.008 and 0.014 , respectively, and not significantly different between other groups.

With the number of CNS diseases, respiratory system diseases, and dermal problems as independent variables, ordinal logistic regression also shows that the number of CNS 
TABLE 3: Comparison of the complications rates between genders.

\begin{tabular}{lccccc}
\hline Factors & $N$ & $\begin{array}{c}\text { Male/N } \\
(\%)\end{array}$ & $\begin{array}{c}\text { Female/N } \\
(\%)\end{array}$ & $p$ & Total/N (\%) \\
\hline $\begin{array}{l}\text { Total } \\
\text { complications }\end{array}$ & & & & 0.592 & \\
& 0 & 520 & 1356 & 0.47 & $1876(99.15)$ \\
& 1 & $3(0.57)$ & $4(0.29)$ & 0.302 & $7(0.37)$ \\
& 2 & $2(0.38)$ & $2(0.15)$ & 0.309 & $4(0.21)$ \\
& 3 & 0 & $1(0.07)$ & 0.723 & $1(0.05)$ \\
& 4 & 0 & $1(0.07)$ & 0.723 & $1(0.05)$ \\
Pressure & 5 & 0 & $3(0.22)$ & 0.377 & $3(0.16)$ \\
ulcer & & 0 & $6(0.44)$ & 0.142 & $6(0.31)$ \\
HAP & & $2(0.38)$ & $7(0.51)$ & 0.524 & $9(0.48)$ \\
UTI & & 0 & $5(0.37)$ & 0.196 & $5(0.26)$ \\
VTE & & 0 & $1(0.07)$ & 0.723 & $1(0.05)$ \\
Constipation & & 0 & $3(0.22)$ & 0.377 & $3(0.16)$ \\
Foot drop & & $3(0.57)$ & $7(0.51)$ & 0.555 & $10(0.53)$ \\
Incision & $2(0.38)$ & $1(0.07)$ & 0.188 & $3(0.16)$ \\
problem & & & & &
\end{tabular}

$N$ : number; UTI: urinary tract infection; VTE: venous thromboembolism; HAP: hospital-acquired pneumonia.

diseases and respiratory system diseases are independent risk factors for the complications number (Table 10).

When all individual potential risk factors are analysed by Pearson's Chi square test, it can be shown that broken skin, skin yellowing and rash, hematocyanosis, hyperpigmentation, cerebral infarction and hemorrhage, dementia, pneumonia, postural hypotension, antidepressant, femoral shaft fracture, osteoarthritis, and anemia are risk factors for the number of the complications (Table 11). However, multivariate analysis shows that only dementia, pneumonia, antidepressant, postural hypotension, and cerebral infarction are independent risk factors for the number of the complications (Table 12).

\section{Discussion}

To our knowledge, this is the first study to explore and describe the relationships between the conditions of comorbidity, medication use, health characteristics, preventive measurements, and the incidences of in-hospital complications. Except lower extremity VTE, constipation, and incision complications, the risk factors for other four complications could be determined using multiple logistic regression model. $\mathrm{Vu}$ et al. reported that men hospitalized due to a fall had a higher comorbidity rate than women [12]. However, our findings were discordant with their results.

4.1. Conscious Status. In Freter et al's study, age was not a risk factor for preoperative delirium, and the strongest association with preoperative delirium was cognitive impairment, substance use, sensory impairment, and wait time for surgery in fall-related hip fracture patients [19]. Our study cannot reveal any independent risk factors for delirium. However, it is shown that antidepressant use is a risk factor for decreased level of conscious status in univariate analysis $(\mathrm{OR}=29.532, p=0.001)$ (Table 4$)$, and multiple logistic regression model has age, nutritional status, dementia, and sensory impairment as independent risk factors for decreased level of conscious status after adjustment for other potential risk factors (Table 5).

4.2. Pressure Ulcer. Kwong et al. showed that bedfast or chairfast patients with stroke were at higher risk for pressure ulcer development [20]. Van Marum et al. also found cerebrovascular accident was a risk factor for decubitus ulcers [21].

In our findings, the cerebral hemorrhage is also an independent risk factor for pressure ulcer development; however, to turn patients over regularly can significantly decrease the risk of pressure ulcer.

Meanwhile, pressure ulcer in univariate analysis was significantly associated with poorer Glasgow coma scale in traumatic brain injury patients [22]. Sayar et al. also found that pressure ulcer development had significant correlation with decreased level of consciousness [23].

However, we cannot find this relationship in the fallrelated fracture patients. The possible reason might be that the fracture patients included in our study all accepted the operative treatment which could reduce LOS in the bed and permit the patients early rehabilitation exercise, mobilization, and comprehensive nursing postoperatively even for the patients with severe consciousness impairment.

4.3. HAP. HAP is one of the most common nosocomial infections. In the present study, two male $(0.38 \%)$ and seven female $(0.51 \%)$ patients developed HAP, and there is no significant difference of HAP incidence between genders.

Sopena et al. reported that patients with malnutrition, anemia, depression of consciousness, and comorbidity had higher incidence of HAP [24]. Zhu et al. proposed that atrial fibrillation was an independent risk factor for HAP [25]. In Guzmán-Herrador et al.'s study, decreased level of consciousness on admission was a risk factor for HAP [26].

In the present study, in addition to atrial fibrillation and decreased level of conscious state we find that cerebral infarction and dementia significantly increase the incidence of HAP.

4.4. UTI. Redder et al. reported that patients with hospitalacquired UTI used indwelling urinary catheters more frequently and had more genitourinary or nervous system diseases than the control group [27]. In the present study, CNS diseases, especially cerebral infarction and dementia, can increase the incidence of hospital-acquired UTI.

In Hagerty et al's study, anemia was a variable significantly associated with hospital-acquired UTI in patients with subarachnoid hemorrhage [28]. In our multiple regression 
TABle 4: Pearson's Chi square test for the risk factors of conscious status.

\begin{tabular}{|c|c|c|c|c|c|c|c|}
\hline \multirow{2}{*}{ Factors } & \multirow{2}{*}{ Yes/no } & \multicolumn{5}{|c|}{ Conscious status } & \multirow{2}{*}{$\chi^{2} / p$} \\
\hline & & 1 & 2 & 3 & 4 & 5 & \\
\hline \multirow{2}{*}{ Intertrochanteric fracture } & No & 1253 & 33 & 0 & 0 & 1 & \multirow{2}{*}{$13.392 / 0.01$} \\
\hline & Yes & 573 & 29 & 1 & 2 & 0 & \\
\hline \multirow{2}{*}{ Cerebral hemorrhage } & No & 1801 & 62 & 1 & 0 & 1 & \multirow{2}{*}{$139.118 /<0.001$} \\
\hline & Yes & 25 & 0 & 0 & 2 & 0 & \\
\hline \multirow{2}{*}{ Cerebral infarction } & No & 1663 & 55 & 0 & 2 & 1 & \multirow{2}{*}{$10.773 / 0.029$} \\
\hline & Yes & 163 & 7 & 1 & 0 & 0 & \\
\hline \multirow{2}{*}{ Dementia } & No & 1808 & 55 & 1 & 2 & 1 & \multirow{2}{*}{$48.886 /<0.001$} \\
\hline & Yes & 18 & 7 & 0 & 0 & 0 & \\
\hline \multirow{2}{*}{ Other CNS diseases } & No & 1708 & 59 & 1 & 2 & 0 & \multirow{2}{*}{$14.982 / 0.005$} \\
\hline & Yes & 118 & 3 & 0 & 0 & 1 & \\
\hline \multirow{2}{*}{ Diabetes } & No & 1502 & 55 & 1 & 0 & 0 & \multirow{2}{*}{$15.945 / 0.003$} \\
\hline & Yes & 324 & 7 & 0 & 2 & 1 & \\
\hline \multirow{2}{*}{ Antidiabetic drug } & No & 1508 & 57 & 1 & 0 & 0 & \multirow{2}{*}{$18.315 / 0.001$} \\
\hline & Yes & 318 & 5 & 0 & 2 & 1 & \\
\hline \multirow{2}{*}{ Antidepressant } & No & 1826 & 61 & 1 & 2 & 1 & \multirow{2}{*}{$29.532 / 0.001$} \\
\hline & Yes & 0 & 1 & 0 & 0 & 0 & \\
\hline \multirow{2}{*}{ Pale skin } & No & 1813 & 58 & 1 & 2 & 1 & \multirow{2}{*}{$22.221 /<0.001$} \\
\hline & Yes & 13 & 4 & 0 & 0 & 0 & \\
\hline \multirow{2}{*}{ Other skin problems } & No & 1059 & 47 & 0 & 0 & 0 & \multirow{2}{*}{$13.473 / 0.009$} \\
\hline & Yes & 767 & 15 & 1 & 2 & 1 & \\
\hline \multirow{2}{*}{ Constipation } & No & 1825 & 61 & 1 & 2 & 1 & \multirow{2}{*}{$13.791 / 0.008$} \\
\hline & Yes & 1 & 1 & 0 & 0 & 0 & \\
\hline \multirow{2}{*}{ Sensory impairment } & No & 1621 & 42 & 1 & 2 & 1 & \multirow{2}{*}{$25.854 /<0.001$} \\
\hline & Yes & 205 & 20 & 0 & 0 & 0 & \\
\hline \multirow{3}{*}{ Nutritional status } & Normal & 1267 & 22 & 1 & 2 & 1 & \multirow{3}{*}{$68 /<0.001$} \\
\hline & Moderate & 522 & 30 & 0 & 0 & 0 & \\
\hline & Malnourished & 37 & 10 & 0 & 0 & 0 & \\
\hline
\end{tabular}

CNS: central nervous system.

TABLE 5: Ordinal logistic regression for conscious status ${ }^{1}$.

\begin{tabular}{lcccc}
\hline Factors & Grade & $\beta$ & $p$ & $95 \%$ CI for $\beta$ \\
\hline Age & & 0.043 & 0.023 & $0.006-0.079$ \\
Nutritional & Normal & -1.935 & $<0.001$ & $-2.82--1.049$ \\
status & Moderate & -1.337 & 0.002 & $-2.188--0.486$ \\
& Malnourished & 0 & & \\
Dementia & No & -2.145 & $<0.001$ & $-3.124--1.165$ \\
Sensory & Yes & 0 & & \\
impairment & No & -0.708 & 0.02 & $-1.305--0.111$ \\
& Yes & 0 & & \\
\hline
\end{tabular}

${ }^{1}$ Hosmer-Lemeshow test, $p<0.001 ; \beta$ : regression coefficient; CI: confidential interval.

model, anemia is also an independent risk factor for hospitalacquired UTI in fall-related fractures patients $(\mathrm{OR}=39.985$, $p=0.034)$. In addition, the preventive measure of bladder irrigation also can increase the incidence of UTI $(\mathrm{OR}=$
14.954, $p=0.009)$, while drinking more water is a protective factor for this complication $(\mathrm{OR}=0.013, p=0.021)$.

4.5. Foot Drop. The complication of foot drop has been shown to be an association with knee dislocation and ligaments' injures and knee arthroplasty surgery [29,30].

In the present study, 10 patients $(0.53 \%)$ developed foot drop during hospitalization. Multiple logistic regression model shows that patients with postural hypotension $(\mathrm{OR}=$ 68.657, $p=0.002)$, dementia $(\mathrm{OR}=19.223, p=0.002)$, broken skin ( $\mathrm{OR}=10.826, p=0.001)$, and cerebral infarction $(\mathrm{OR}=7.165, p=0.011)$ are at a higher risk for foot drop development. The possible reason might be the longer time in bed for these patients. However, we cannot find the differences of hospital length of stay or grade 1 nursing between the patients with these comorbidities and without them.

4.6. Complications Number. Harvey et al. reported that patients with dementia were disproportionately represented in injury-related hospitalizations, experienced longer hospital LOS, and had poorer outcomes [31]. Other studies 
TABLE 6: Univariate and multivariate analysis for risk factors of pressure ulcer.

\begin{tabular}{|c|c|c|c|c|c|c|}
\hline \multirow{2}{*}{ Factors } & \multicolumn{3}{|c|}{ Univariate analysis } & \multicolumn{3}{|c|}{ Multivariate logistic regression $^{1}$} \\
\hline & OR & $p$ & $95 \% \mathrm{CI}$ & OR & $p$ & $95 \% \mathrm{CI}$ \\
\hline Femoral shaft fracture & 7.92 & 0.005 & $1.435-43.704$ & 23.640 & 0.023 & $1.541-362.673$ \\
\hline Broken skin & 12.597 & $<0.001$ & $2.27-69.904$ & 14.324 & 0.07 & $0.806-254.494$ \\
\hline Cerebral hemorrhage & 14.308 & 0.002 & $1.615-126.778$ & 555.49 & 0.002 & $10.017-30805.37$ \\
\hline Dementia & 40.5 & $<0.001$ & $7.063-232.241$ & 145.3 & 0.003 & $5.711-3697.031$ \\
\hline Turning over regularly & 0.036 & $<0.001$ & $0.006-0.201$ & 0.039 & 0.017 & $0.003-0.562$ \\
\hline Skin yellowing & 34.091 & $<0.001$ & $3.676-316.197$ & & & \\
\hline Skin rash & 20.756 & $<0.001$ & $2.308-186.691$ & & & \\
\hline Other skin problems & 0.995 & 0.039 & $0.99-0.999$ & & & \\
\hline Hypertension & 0.994 & 0.034 & $0.99-0.999$ & & & \\
\hline Anemia & 14.308 & 0.002 & $1.615-126.778$ & & & \\
\hline
\end{tabular}

${ }^{1}$ Hosmer-Lemeshow test, $p=0.217$; OR: odds ratio; CI: confidential interval.

TABLE 7: Univariate and multivariate analysis for risk factors of hospital-acquired pneumonia.

\begin{tabular}{|c|c|c|c|c|c|c|}
\hline \multirow{2}{*}{ Factors } & \multicolumn{3}{|c|}{ Univariate analysis } & \multicolumn{3}{|c|}{ Multivariate logistic regression $^{1}$} \\
\hline & OR & $p$ & $95 \% \mathrm{CI}$ & OR & $p$ & $95 \% \mathrm{CI}$ \\
\hline Atrial fibrillation & 95.789 & 0.004 & $4.184-2192.782$ & 95.789 & 0.007 & $6.42-1890.43$ \\
\hline Cerebral infarction & 5.104 & 0.011 & $1.265-20.594$ & 25.791 & 0.001 & $3.728-178.45$ \\
\hline Dementia & 23.106 & $<0.001$ & $4.553-117.267$ & 8.545 & 0.007 & $1.795-40.678$ \\
\hline Conscious state & 1.138 & 0.031 & $1.017-2.139$ & 1.850 & 0.007 & $0.511-3.189$ \\
\hline Antihypertensive drugs & 0.992 & 0.011 & $0.987-0.997$ & & & \\
\hline
\end{tabular}

${ }^{1}$ Hosmer-Lemeshow test, $p=0.629$; OR: odds ratio; CI: confidential interval.

TABLE 8: Univariate and multivariate analysis of risk factors for urinary tract infection.

\begin{tabular}{|c|c|c|c|c|c|c|}
\hline \multirow{2}{*}{ Factors } & \multicolumn{3}{|c|}{ Univariate analysis } & \multicolumn{3}{|c|}{ Multivariate logistic regression ${ }^{1}$} \\
\hline & OR & $p$ & $95 \% \mathrm{CI}$ & OR & $p$ & $95 \% \mathrm{CI}$ \\
\hline Cerebral infarction & 6.777 & 0.016 & $1.125-40.842$ & 88.807 & 0.026 & $1.688-4671.192$ \\
\hline Dementia & 54.029 & $<0.001$ & $8.617-338.782$ & 1017.668 & 0.002 & $12.626-82027.991$ \\
\hline Anemia & 17.894 & $<0.001$ & $1.933-165.622$ & 39.985 & 0.034 & $1.317-1213.702$ \\
\hline Drinking more water & 0.043 & $<0.001$ & $0.005-0.401$ & 0.013 & 0.021 & $0.000-0.515$ \\
\hline Bladder irrigation & 25.716 & $<0.001$ & $4.251-155.588$ & 14.954 & 0.009 & $1.983-112.788$ \\
\hline Skin yellowing & 42.636 & $<0.001$ & $4.405-412.712$ & & & \\
\hline Skin rash & 25.958 & $<0.001$ & $2.764-243.819$ & & & \\
\hline Broken skin & 16.806 & $<0.001$ & $2.765-102.139$ & & & \\
\hline Cerebral hemorrhage & 17.894 & $<0.001$ & $1.933-165.622$ & & & \\
\hline
\end{tabular}

${ }^{1}$ Hosmer-Lemeshow test, $p=0.652$; OR: odds ratio; CI: confidential interval.

TABLE 9: Univariate and multivariate analysis for risk factors of foot drop.

\begin{tabular}{lcccccc}
\hline \multirow{2}{*}{ Factors } & \multicolumn{3}{c}{ Univariate analysis } & \multicolumn{3}{c}{ Multivariate logistic regression $^{1}$} \\
& OR & $p$ & $95 \%$ CI & OR & $p$ & $95 \%$ CI \\
\hline Broken skin & 17.257 & $<0.001$ & $4.762-62.534$ & 10.826 & 0.001 & $2.640-44.388$ \\
Cerebral infarction & 4.372 & 0.02 & $1.12-17.066$ & 7.165 & 0.011 & $1.567-32.77$ \\
Dementia & 20.207 & $<0.001$ & $4.067-100.396$ & 19.223 & 0.002 & $2.915-126.765$ \\
Postural hypotension & 52.167 & $<0.001$ & $5.298-513.636$ & 68.657 & 0.002 & $4.801-981.923$ \\
\hline
\end{tabular}

${ }^{1}$ Hosmer-Lemeshow test, $p=0.398$; OR: odds ratio; CI: confidential interval. 
TABLE 10: Univariate and multivariate analysis for risk factors of the number of complications.

\begin{tabular}{|c|c|c|c|c|c|c|c|c|c|c|c|}
\hline \multirow{3}{*}{ Comorbidities } & \multirow{3}{*}{$N$} & \multicolumn{7}{|c|}{ Univariate analysis } & \multicolumn{3}{|c|}{ Ordinal logistic regression $^{1}$} \\
\hline & & \multicolumn{6}{|c|}{ Complications } & \multirow{2}{*}{$\chi^{2} / p$} & \multirow{2}{*}{$\beta$} & \multirow{2}{*}{$p$} & \multirow{2}{*}{$95 \%$ CI for $\beta$} \\
\hline & & 0 & 1 & 2 & 3 & 4 & 5 & & & & \\
\hline \multirow{4}{*}{ CNS diseases } & 0 & 1455 & 3 & 3 & 0 & 0 & 0 & $34.236 / 0.003$ & 14.785 & $<0.001$ & $13.157-16.412$ \\
\hline & 1 & 375 & 3 & 1 & 1 & 1 & 2 & & 16.432 & $<0.001$ & $14.853-18.011$ \\
\hline & 2 & 40 & 1 & 0 & 0 & 0 & 1 & & 17.255 & & $17.255-17.255$ \\
\hline & 3 & 6 & 0 & 0 & 0 & 0 & 0 & & 0 & & \\
\hline \multirow{2}{*}{ Respiratory system diseases } & 0 & 1808 & 4 & 4 & 1 & 1 & 3 & $30.072 /<0.001$ & -1.788 & 0.007 & $-3.079--0.498$ \\
\hline & 1 & 68 & 3 & 0 & 0 & 0 & 0 & & 0 & & \\
\hline \multirow{4}{*}{ Dermal problems } & 0 & 468 & 1 & 2 & 1 & 1 & 0 & $27.808 / 0.023$ & & & \\
\hline & 1 & 1378 & 6 & 2 & 0 & 0 & 2 & & & & \\
\hline & 2 & 29 & 0 & 0 & 0 & 0 & 1 & & & & \\
\hline & 3 & 1 & 0 & 0 & 0 & 0 & 0 & & & & \\
\hline
\end{tabular}

${ }^{1}$ Hosmer-Lemeshow test for CNS diseases and respiratory system diseases, $p=0.005$ and 0.022 , respectively; $\beta$ : regression coefficient; CNS: central nervous system.

TABLE 11: Univariate analysis for risk factors of the number of complications.

\begin{tabular}{lcc}
\hline \multirow{2}{*}{ Factors } & \multicolumn{2}{c}{ Univariate analysis } \\
& $\chi^{2}$ & $p$ \\
\hline Cerebral infarction & 22.378 & $<0.001$ \\
Dementia & 98.549 & $<0.001$ \\
Pneumonia & 32.099 & $<0.001$ \\
Postural hypotension & 52.423 & $<0.001$ \\
Antidepressant & 88.58 & $<0.001$ \\
Broken skin & 38.418 & $<0.001$ \\
Femoral shaft fracture & 17.387 & 0.004 \\
Skin yellowing & 51.049 & $<0.001$ \\
Hematocyanosis & 28.338 & $<0.001$ \\
Skin rash & 31.709 & $<0.001$ \\
Hyperpigmentation & 18.587 & 0.002 \\
Cerebral hemorrhage & 21.921 & 0.001 \\
Osteoarthritis & 14.201 & 0.014 \\
Anemia & 21.921 & 0.001 \\
\hline
\end{tabular}

also showed that delirium was associated with several complications, including longer hospital LOS, more function and cognition impairment, increased risk of nursing home placement, and even death, in elderly orthopedic patients [32-35].

In our findings, dementia is also shown to be the strongest association with all the types of complications. However, we cannot find that hospital LOS is longer in dementia patients than patients without it. Besides dementia, cerebral infarction and broken skin are also independent risk factors for four and three types of complications, respectively. However, multiple logistic regression model only has pneumonia, postural hypotension, and antidepressant as independent risk factors for the complications number rather than broken skin.
4.7. Limitations. This study has several limitations. First, we cannot determine the risk factors for lower extremity VTE, constipation, and incision complications based on the present database. The possible reason might be that the incidences of the lower extremity VTE, constipation, and incision complications were too low with $0.05 \%, 0.16 \%$, and $0.16 \%$, respectively. Second, most of the preventive measures of the complications analysed in this study cannot increase or reduce the incidences of in-hospital complications analysed, except the self-contradictory impact of bladder irrigation and drinking more water on UTI. Third, no control group but only fall-related fracture patients are included and analysed. Thus, the risk factors for fall-related fractures cannot be explored and analysed in this study. Fourth, no long-term follow-up results, such as internal fixation failure or death, and the corresponding incidences and risks factors are collected and determined.

\section{Conclusion}

Different combinations of comorbidity, medication use, and preventive measurements were related to the number and pattern of in-hospital complications of patients with fallrelated fractures. Dementia emerged as the most important risk factor for these complications, while most of the preventive measurements could not reduce the incidence of the inhospital complications. Continued studies are still warranted to verify these associations and determine how to incorporate consideration of comorbidity into preventive assessments of in-hospital complications of fall-related fractures.

\section{Disclosure}

This research received no specific grant from any funding agency in the public, commercial, or not-for-profit sectors.

\section{Competing Interests}

The authors declare that they have no conflict of interests. 
TABLE 12: Ordinal logistic regression for risk factors of the number of complications.

\begin{tabular}{|c|c|c|c|c|}
\hline \multirow{2}{*}{ Factors } & \multirow{2}{*}{ Yes/no } & \multicolumn{3}{|c|}{ Ordinal logistic regression $^{1}$} \\
\hline & & $\beta$ & $p$ & $95 \%$ CI for $\beta$ \\
\hline \multirow{2}{*}{ Cerebral infarction } & No & -1.610 & \multirow{2}{*}{0.028} & \multirow{2}{*}{$-3.045--0.175$} \\
\hline & Yes & 0 & & \\
\hline \multirow{2}{*}{ Dementia } & No & -3.232 & \multirow{2}{*}{$<0.001$} & \multirow{2}{*}{$-4.936--1.527$} \\
\hline & Yes & 0 & & \\
\hline \multirow{2}{*}{ Pneumonia } & No & -2.162 & \multirow{2}{*}{0.007} & \multirow{2}{*}{$-3.741--0.582$} \\
\hline & Yes & 0 & & \\
\hline \multirow{2}{*}{ Postural hypotension } & No & -3.495 & \multirow{2}{*}{0.024} & \multirow{2}{*}{$-6.530--0.460$} \\
\hline & Yes & 0 & & \\
\hline \multirow{2}{*}{ Antidepressant } & No & -4.251 & \multirow{2}{*}{0.007} & \multirow{2}{*}{$-7.358--1.144$} \\
\hline & Yes & 0 & & \\
\hline
\end{tabular}

${ }^{1}$ Hosmer-Lemeshow test, $p<0.001 ; \beta$ : regression coefficient; CI: confidential interval.

\section{Authors' Contributions}

Jing Wang and Yuan Gao collected the data and drafted the manuscript. Hong-Ying $\mathrm{Pi}$ and Meng-Meng $\mathrm{Hu}$ revised the manuscript. Hong-Ying Pi and Yuan Gao conceived the study and participated in its design and coordination. Statistical analysis was done by Pei-Pei Peng and Dan Nie. All authors read and approved the final manuscript.

\section{References}

[1] L. Z. Rubenstein and K. R. Josephson, "Falls and their prevention in elderly people: what does the evidence show?" Medical Clinics of North America, vol. 90, no. 5, pp. 807-824, 2006.

[2] P. Kannus, H. Sievänen, M. Palvanen, T. Järvinen, and J. Parkkari, "Prevention of falls and consequent injuries in elderly people," The Lancet, vol. 366, no. 9500, pp. 1885-1893, 2005.

[3] R. M. Ravindran and V. R. Kutty, "Risk factors for fallrelated injuries leading to hospitalization among communitydwelling older persons: a hospital-based case-control study in Thiruvananthapuram, Kerala, India," Asia-Pacific Journal of Public Health, vol. 28, no. 1, pp. 70S-76S, 2016.

[4] K. M. Sibley, J. Voth, S. E. Munce, S. E. Straus, and S. B. Jaglal, "Chronic disease and falls in community-dwelling Canadians over 65 years old: a population-based study exploring associations with number and pattern of chronic conditions," $B M C$ Geriatrics, vol. 14, article no. 22, 2014.

[5] W. Muangpaisan, S. Suwanpatoomlerd, V. Srinonprasert, W. Sutipornpalangkul, A. Wongprikron, and P. Assantchai, "Causes and course of falls resulting in hip fracture among elderly Thai patients," Journal of the Medical Association of Thailand, vol. 98, no. 3, pp. 298-305, 2015.

[6] B. Leavy, L. Byberg, K. Michaëlsson, H. Melhus, and A. C. Åberg, "The fall descriptions and health characteristics of older adults with hip fracture: a mixed methods study," $B M C$ Geriatrics, vol. 15, no. 1, article 40, 2015.

[7] T. S. H. Jørgensen, A. H. Hansen, M. Sahlberg et al., "Nationwide time trends and risk factors for in-hospital falls-related major injuries," International Journal of Clinical Practice, vol. 69, no. 6, pp. 703-709, 2015.

[8] S.-I. Toyabe, "Development of a risk assessment tool to predict fall-related severe injuries occurring in a hospital," Global Journal of Health Science, vol. 6, no. 5, pp. 70-80, 2014.
[9] K.-Y. Cheng, W.-C. Lin, W.-N. Chang et al., "Factors associated with fall-related fractures in Parkinson's disease," Parkinsonism and Related Disorders, vol. 20, no. 1, pp. 88-92, 2014.

[10] F. Nilson, S. Moniruzzaman, and R. Andersson, "Fall-related fracture trends among elderly in Sweden-exoring transitions among hospitalized cases," Journal of Safety Research, vol. 45, pp. 141-145, 2013.

[11] W. L. Watson and R. Mitchell, "Conflicting trends in fall-related injury hospitalisations among older people: variations by injury type," Osteoporosis International, vol. 22, no. 10, pp. 2623-2631, 2011.

[12] T. Vu, C. F. Finch, and L. Day, "Patterns of comorbidity in community-dwelling older people hospitalised for fall-related injury: a cluster analysis," BMC Geriatrics, vol. 11, article no. 45, 2011.

[13] H. Shimada, M. Suzukawa, T. Ishizaki, K. Kobayashi, H. Kim, and T. Suzuki, "Relationship between subjective fall risk assessment and falls and fall-related fractures in frail elderly people," BMC Geriatrics, vol. 11, article 40, 2011.

[14] Medical Advisory Secretariat, "Prevention of falls and fallrelated injuries in community-dwelling seniors: an evidencebased analysis," Ontario Health Technology Assessment Series, vol. 8, no. 2, pp. 1-78, 2008.

[15] C. Wallace, G. E. Reiber, J. LeMaster et al., "Incidence of falls, risk factors for falls, and fall-related fractures in individuals with diabetes and a prior foot ulcer," Diabetes Care, vol. 25, no. 11, pp. 1983-1986, 2002.

[16] M. E. Tinetti and C. Kumar, "The patient who falls: 'it's always a trade-off"' The Journal of the American Medical Association, vol. 303, no. 3, pp. 258-266, 2010.

[17] K. Kallin, J. Jensen, L. L. Olsson, L. Nyberg, and Y. Gustafson, "Why the elderly fall in residential care facilities, and suggested remedies," Journal of Family Practice, vol. 53, no. 1, pp. 41-52, 2004.

[18] M. C. Murphy, C. N. Brooks, S. A. New, and M. L. Lumbers, “The use of the Mini-Nutritional Assessment (MNA) tool in elderly orthopaedic patients," European Journal of Clinical Nutrition, vol. 54, no. 7, pp. 555-562, 2000.

[19] S. Freter, M. Dunbar, K. Koller, C. MacKnight, and K. Rockwood, "Risk of pre-and post-operative delirium and the delirium elderly at risk (DEAR) tool in hip fracture patients," Canadian Geriatrics Journal, vol. 18, no. 4, pp. 212-216, 2015. 
[20] E. W.-Y. Kwong, S. M.-C. Pang, G. H. Aboo, and S. S.-M. Law, "Pressure ulcer development in older residents in nursing homes: influencing factors," Journal of Advanced Nursing, vol. 65, no. 12, pp. 2608-2620, 2009.

[21] R. J. Van Marum, J. H. Meijer, M. E. Ooms, P. J. Kostense, J. T. Van Eijk, and M. W. Ribbe, "Relationship between internal risk factors for development of decubitus ulcers and the blood flow response following pressure load," Angiology, vol. 52, no. 6, pp. 409-416, 2001.

[22] M. Dhandapani, S. Dhandapani, M. Agarwal, and A. K. Mahapatra, "Pressure ulcer in patients with severe traumatic brain injury: significant factors and association with neurological outcome," Journal of Clinical Nursing, vol. 23, no. 7-8, pp. 11141119, 2014.

[23] S. Sayar, S. Turgut, H. Doğan et al., "Incidence of pressure ulcers in intensive care unit patients at risk according to the Waterlow scale and factors influencing the development of pressure ulcers," Journal of Clinical Nursing, vol. 18, no. 5, pp. 765-774, 2009.

[24] N. Sopena, E. Heras, I. Casas et al., "Risk factors for hospitalacquired pneumonia outside the intensive care unit: a casecontrol study," American Journal of Infection Control, vol. 42, no. 1, pp. 38-42, 2014.

[25] J. Zhu, X. Zhang, G. Shi, K. Yi, and X. Tan, "Atrial fibrillation is an independent risk factor for hospital-acquired pneumonia," PLOS ONE, vol. 10, no. 7, Article ID e0131782, 2015.

[26] B. Guzmán-Herrador, C. D. Molina, M. F. Allam, and R. F. Navajas, "Independent risk factors associated with hospitalacquired pneumonia in an adult ICU: 4-year prospective cohort study in a university reference hospital," Journal of Public Health, vol. 38, no. 2, pp. 378-383, 2016.

[27] J. D. Redder, R. A. Leth, and J. K. Møller, "Analysing risk factors for urinary tract infection based on automated monitoring of hospital-acquired infection," Journal of Hospital Infection, vol. 92, no. 4, pp. 397-400, 2016.

[28] T. Hagerty, L. Kertesz, J. M. Schmidt et al., "Risk factors for catheter-associated urinary tract infections in critically Ill patients with subarachnoid hemorrhage," Journal of Neuroscience Nursing, vol. 47, no. 1, pp. 51-54, 2015.

[29] G. Cush and K. Irgit, "Drop foot after knee dislocation: evaluation and treatment," Sports Medicine and Arthroscopy Review, vol. 19, no. 2, pp. 139-146, 2011.

[30] J. W. Pritchett, "Lumbar decompression to treat foot drop after hip arthroplasty," Clinical Orthopaedics and Related Research, no. 303, pp. 173-177, 1994.

[31] L. Harvey, R. Mitchell, H. Brodaty, B. Draper, and J. Close, "The influence of dementia on injury-related hospitalisations and outcomes in older adults," Injury, vol. 47, no. 1, pp. 226-234, 2016.

[32] M. Krogseth, T. B. Wyller, K. Engedal, and V. Juliebø, "Delirium is a risk factor for institutionalization and functional decline in older hip fracture patients," Journal of Psychosomatic Research, vol. 76, no. 1, pp. 68-74, 2014.

[33] A. Morandi, D. Davis, D. M. Fick et al., "Delirium superimposed on dementia strongly predicts worse outcomes in older rehabilitation inpatients," Journal of the American Medical Directors Association, vol. 15, no. 5, pp. 349-354, 2014.

[34] M. G. Kat, J. F. de Jonghe, R. Vreeswijk et al., "Mortality associated with delirium after hip-surgery: A 2-year Follow-up Study," Age and Ageing, vol. 40, no. 3, pp. 312-318, 2011.

[35] J. Witlox, L. S. M. Eurelings, J. F. M. de Jonghe, K. J. Kalisvaart, P. Eikelenboom, and W. A. van Gool, "Delirium in elderly patients and the risk of postdischarge mortality, institutionalization, and dementia: a meta-analysis," The Journal of the American Medical Association, vol. 304, no. 4, pp. 443-451, 2010. 


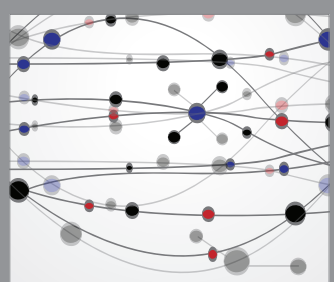

The Scientific World Journal
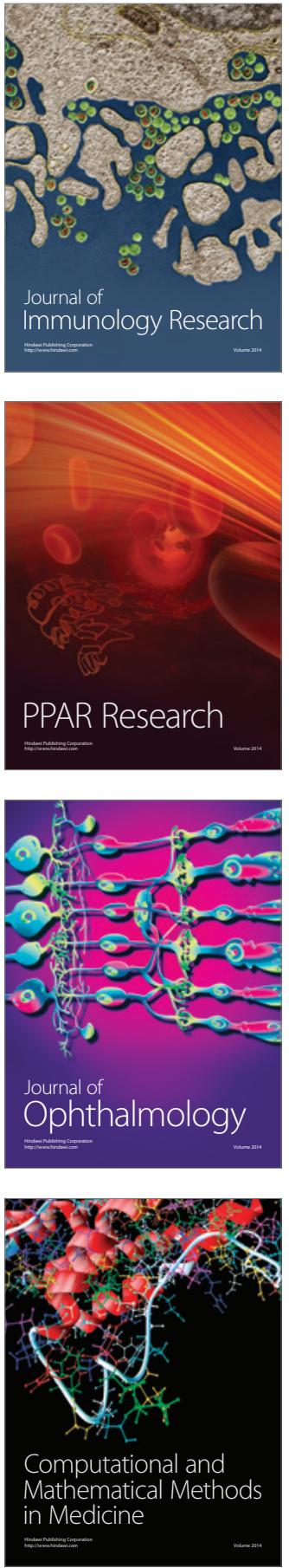

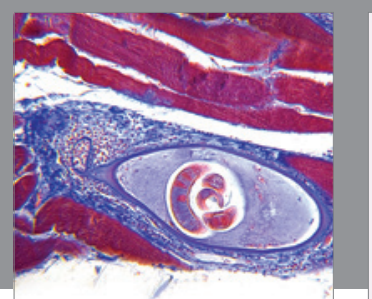

Gastroenterology Research and Practice

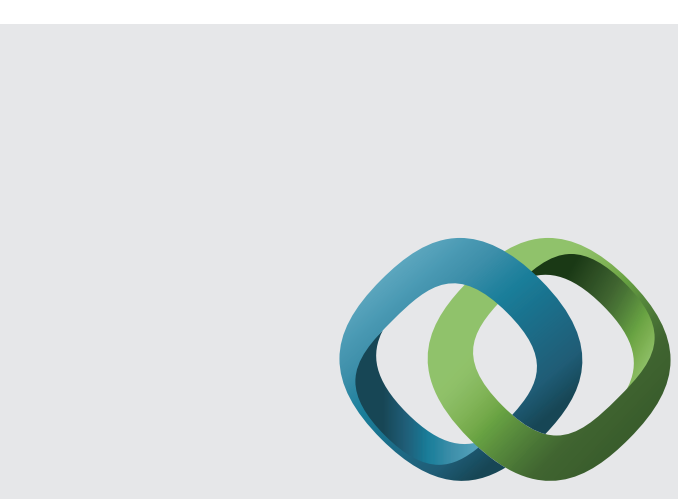

\section{Hindawi}

Submit your manuscripts at

http://www.hindawi.com
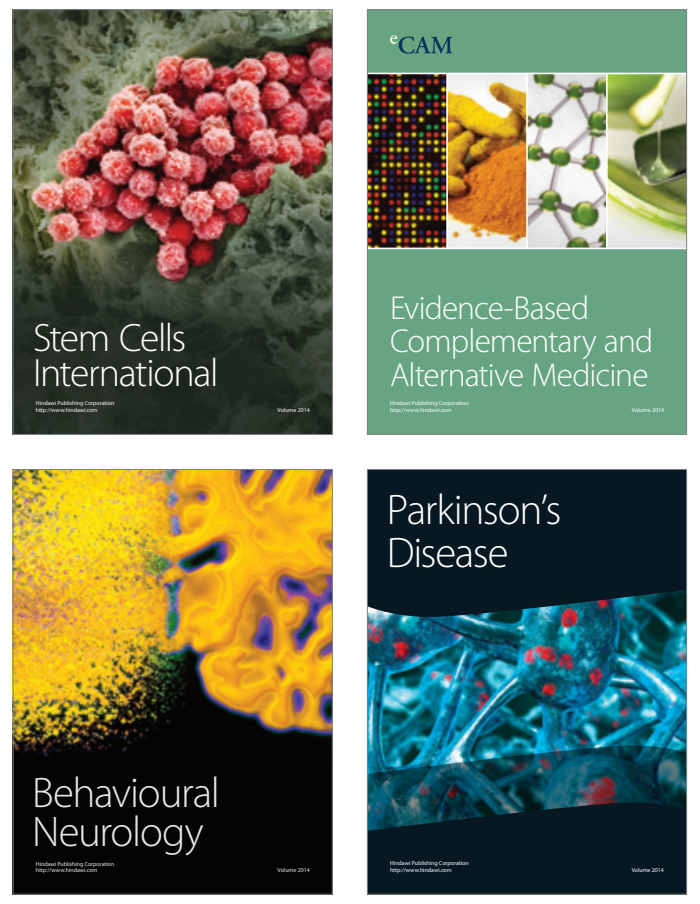
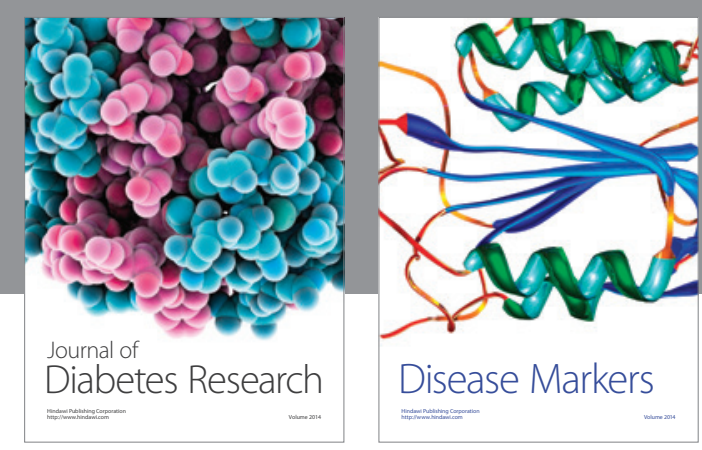

Disease Markers
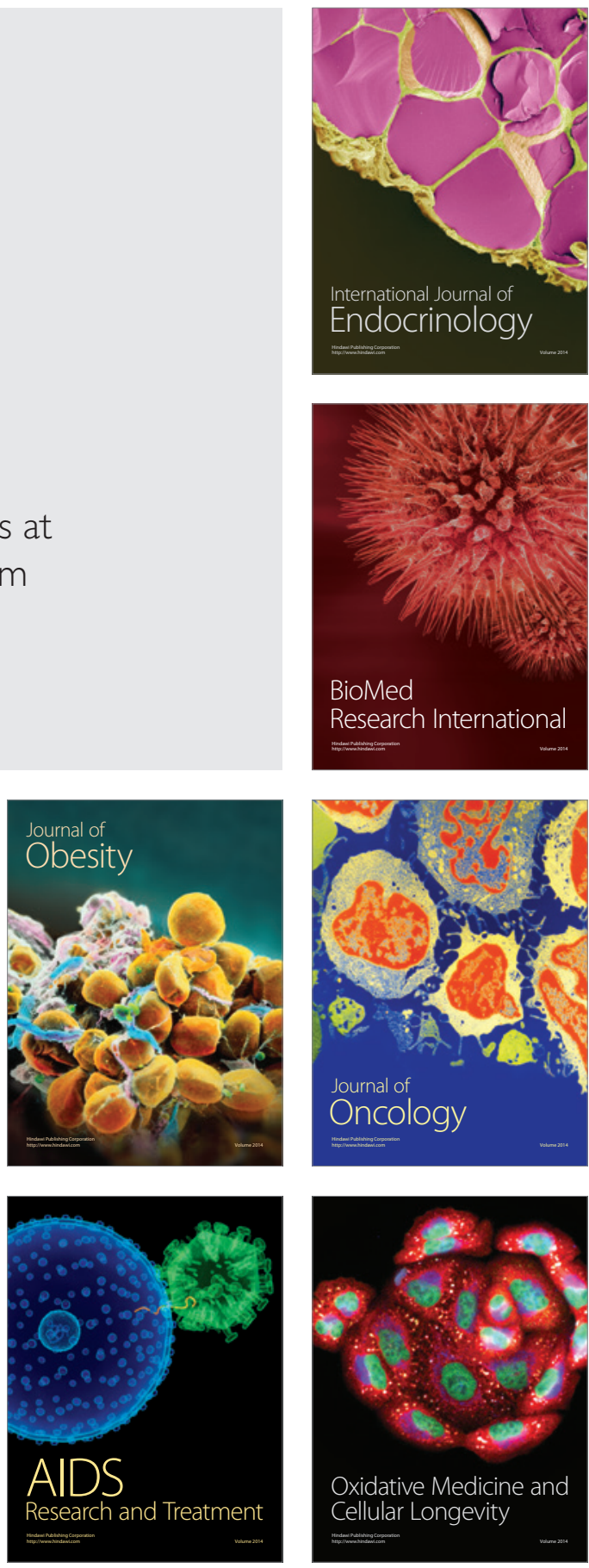